\title{
Oscars of science reward photonics
}

Two visionaries of optical lattice clock technology have received recognition in this year's Breakthrough Prizes.

Fi: or the very first time, the Breakthrough

Prize in Fundamental Physics has been awarded to pioneers working in the area of photonics. Hidetoshi Katori from the University of Tokyo/RIKEN in Japan and Jun Ye from the University of Colorado/ NIST in the US are co-recipients of the 2022 prize for their contributions to the invention and development of the optical lattice clock ${ }^{1}$. They will equally share the US\$3 million prize, each receiving US\$1.5 million.

Optical lattice clocks have evolved to become the world's most precise timekeepers, with a precision of around $10^{-19}$ being set in $2018^{2}$. Indeed, there is talk of them being used in the near-future to provide a new, more precise reference standard for the second, the fundamental unit of time, as per the international system of units.

At present, that responsibility rests with the caesium atomic clock, with 1 second being defined as 9,192,631,770 periods of the microwave radiation emitted between two hyperfine levels in the ground state of an atom of caesium-133, achieving a precision of the order of $10^{-16}$. However, it may not be long before the strontium optical lattice clock - operating with a red, visible transition at a frequency of $430 \mathrm{THz}-$ takes over, given its astonishing accuracy. Such a clock keeps accurate time to $\pm 1 \mathrm{~s}$ in 15 billion years.

Lattice clocks take their name from the use of a periodic potential (the lattice), in one, two or even three dimensions to hold thousands of ultracold (temperatures of $\sim 15 \mathrm{nK}$ ) strontium atoms, almost perfectly still in unison, allowing the atomic ensemble to exhibit a quantum coherence of order of $15 \mathrm{~s}$ and emit an almost perfectly pure clock frequency. Creating the lattice with light with the so-called magic wavelength minimizes any undesirable perturbations that would degrade the precision of the transition frequency.

In this issue of Nature Photonics, we celebrate the news of the prize with in-depth interviews with the two winners, Katori and Ye, who each describe how they first got started with trying to make optical lattice clocks, the challenges involved and their potential applications.

And the applications are tantalizing. The incredible precision of optical clocks makes it possible to probe and test the temporal behaviour of the fundamental

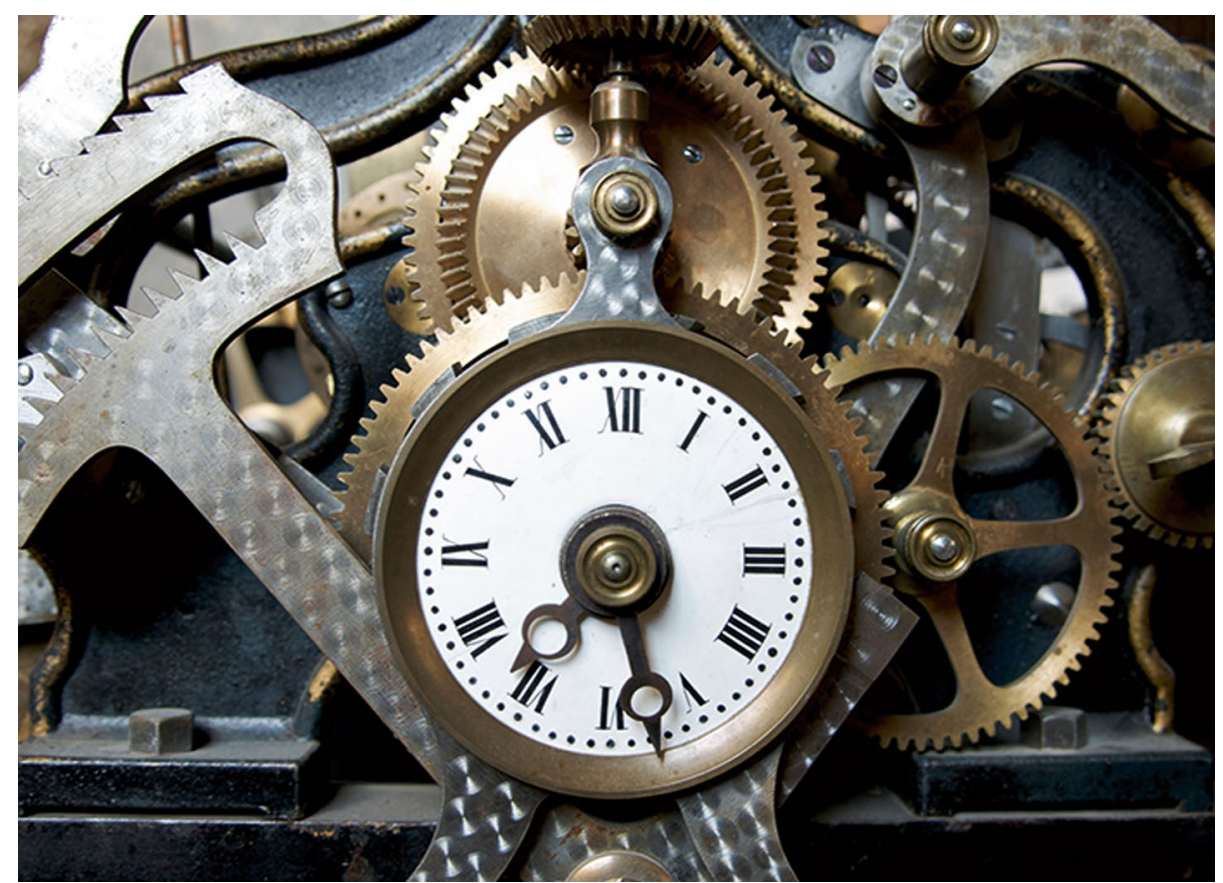

Credit: incamerastock / Alamy Stock Photo

laws of physics, such as Einstein's theories of general relativity and special relativity, with a new level of scrutiny. Such clocks can also be used to investigate if the fundamental physical constants are static or have a dynamic nature. Furthermore, they make it possible to perform new exciting forms of metrology, such as relativistic geodesy ${ }^{3}$, whereby the fact that the timekeeping of clocks varies if they are at different heights (due to different gravitational potentials) means that clocks can be used to perform highly sensitive surveying. Lastly, clocks play a valuable role in global positioning and navigation systems.

While continuing to push the limits of clock precision to the level of $10^{-20}$ and $10^{-21}$ and beyond is of course important using technologies such as quantum squeezed states, perhaps another equally important endeavour is finding ways to make clocks smaller, more cost-effective and robust so that they can be more widely deployed. Integrated optics may play an important role here with creating a convenient, scalable platform. Chip-scale microwave clocks, as developed by John Kitchin at NIST, already exist and have been commercialized, albeit with a lower level of precision, in the region of $10^{-11}$ (ref. ${ }^{4}$ ). Kitchin has more recently developed an optical rubidium atomic clock in an integrated optical chip with a stability of $1.7 \times 10^{-13}$ (ref. $^{4}$ ).

Now in its tenth year, the Breakthrough Prize, commonly known as the Oscars of science, was first established in 2012. Annual prizes exist in the fields of Fundamental Physics, Mathematics, and the Life Sciences. Previous winners of the Fundamental Physics prize include the members of the Event Horizon Telescope Collaboration, Charles Kane and Eugene Mele for their work in topology, the WMAP team for their work on mapping the Universe, and others. Nominations for next year's prize will open shortly and close in April.

Published online: 20 December 2021 https://doi.org/10.1038/s41566-021-00941-5

\footnotetext{
References

1. https://breakthroughprize.org/News/65

2. Marti, G. E. et al. Phys. Rev. Lett. 120, 103201 (2018)

. Takamoto, M. et al. Nat. Photon. 14, 411-415 (2020).

Newman, Z. L. et al. Optica 6, 680-685 (2020).
} 